\title{
Multilevel strategies for parametric shape optimization in aerodynamics
}

\author{
Badr Abou El Majd - Jean-Antoine Désidéri \\ Régis Duvigneau
}

* Opale Project Team, INRIA, 2004 Route des Lucioles, BP 93

F-06902 Sophia-Antipolis cedex

\{Badr.AbouElMajd; Jean-Antoine.Desideri ; Regis.Duvigneau\}@sophia.inria.fr

ABSTRACT. The essential numerical features of multilevel strategies developed for parametric shape optimization are reviewed. These methods employ nested parameterization supports of either shape, or shape deformation, and the classical process of degree elevation resulting in exact geometrical data transfer from coarse to fine representations. The algorithms mimick classical multigrid strategies and are found very effective in terms of convergence acceleration. In particular, for a drag reduction problem involving a three-dimensional Eulerian transonic flow simulated by an unstructured-grid finite-volume method, the complete algorithm is found to be noticeably superior to the natural algorithm simply based on progressive degree elevation.

RÉSUMÉ. On décrit les principaux éléments numériques de stratégies multiniveaux développées pour l'optimisation paramétrique de forme. Ces méthodes s'appuient sur la construction de supports emboîtés de paramétrisation de la forme, ou de la déformation de forme, et sur le processus classique d'élévation du degré qui permet le transfert géométrique exact de la représentation grossière à la fine. Les algorithmes miment les stratégies multigrilles classiques et se révèlent efficaces pour accélérer la convergence. En particulier, pour un problème de réduction de traînée régi par un écoulement transsonique eulérien tridimensionnel simulé par une méthode de volumes-finis en maillage non structuré, l'algorithme complet s'est révélé supérieur à l'algorithme naturel s'appuyant simplement sur l'élévation progressive du degré.

KEYWORDS: optimum shape design, compressible aerodynamics, finite-volume methods, shape parameterization, multilevel algorithms.

MOTS-CLÉS : conception optimale de forme, aérodynamique compressible, volumes-finis, paramétrisation de forme, algorithmes multiniveaux.

DOI:10.3166/REMN.17.149-168 @ 2008 Lavoisier, Paris

REMN - 17/2008. Shape design in aerodynamics, pages 149 to 168 


\section{Introduction, hierarchical approaches in analysis and design}

In recent years, the achievements in Computational Fluid Dynamics (CFD) have opened the way to optimization, mostly shape-optimization, and design in compressible aerodynamics and coupled disciplines. Aerodynamic design is a field at the interface between several classical disciplines and in which numerical simulations require assembling a number of technical elements either related to geometry (mesh and/or CAD), solvers for a detailed analysis of the physical situations, optimizers to devise a convergent loop permitting to optimize, or simply improve one or more performance indices, or software engineering. Thus, a number of acute topics in numerical analysis, multi-disciplinary engineering and software development are raised by these problems. These include non exhaustively the following:

- multi-disciplinary-optimization (MDO) or concurrent engineering from both technical and organizational viewpoints (design platforms);

- treatment of multi-criterion problems: identification of Pareto fronts, dynamic games;

- model reduction, meta-models and sensitivity analysis (response surface, Kriging, artificial neural networks);

- cost-efficient optimizers (BFGS, SQP, etc.);

- robust optimizers for multi-modal or non-differentiable optimization problems (evolutionary computing: genetic or particle-swarm algorithms, etc.); and hybridization with deterministic optimizers;

- software engineering: automatic-differentiation, code coupling; parallel and asynchronous algorithms;

- CAD and parameterization in shape optimization, and interface with mesh generation and adaptation.

Hence the field of optimum-shape design in aerodynamics is unsurprisingly very active, as the program of several recent courses and workshops demonstrate (Haase et al., 2005; Winter-Althaus et al., 2006; Périaux et al., 2006).

The present contribution relates to the development of cost-efficient optimization strategies relying on multilevel shape parameterization.

Our developments focus on the definition, mathematical analysis and experimentation of numerical methods for shape optimization for applications in which the cost functional evaluation relies on the prior solution of a complex set of partial-differential equations (PDEs), such as those governing compressible aerodynamics (e.g. the Euler equations), or related coupled disciplines such as structural mechanics (e.g. elasticity), or electromagnetics (e.g. the Maxwell equations). These PDEs are very commonly solved by Finite Elements or Volumes, by techniques that, although becoming increasingly standard, are still very costly when the accuracy requirement is high.

The important development of multigrid methods in recent years has demonstrated that such techniques not only permit to accelerate the iterative convergence of solution 
procedures, but also have the more general merit of a better control on grid dependency and convergence. In fact, a linear convergence rate can be demonstrated assuming adequate iterative termination criteria are devised, that is, when the grid-convergence control is enforced properly.

Thus, our efforts are mostly concentrated on improving the convergence rate of numerical procedures both from the viewpoint of cost-efficiency and accuracy, with the perspective of reducing the design cost, but also of mastering the election and control of the design parameters, geometrical ones in particular, in a more rational way, perhaps supported by error estimates.

Technically, our efforts tend to contribute to the following challenges:

- construct multilevel (multi-scale) shape-optimization algorithms;

- identify critical algorithmic ingredients (transfer operators, smoothers);

- evaluate efficiency, theorize convergence via error estimates or an appropriate modal analysis.

We note that prior to us, several other authors have applied multilevel principles in shape optimization. In particular, (Jameson, 1988; Jameson et al., 1998) has used a multigrid method to solve both flow and continuous-adjoint equations with great success. Ta' asan (Arian et al., 1995) introduced the concept of one-shot methods in which these equations are solved in a simultaneous multigrid iteration. Lewis and Nash in (Lewis et al., 2000; Lewis et al., 2005) have introduced a multigrid approach to differential systems in which different approximations to the optimization problem associated with a hierarchy of meshes are coordinated according to a multigrid strategy. Their algorithm is a nonlinear programming adaptation of the multigrid construction. We also point out the recent theoretical review of such methods by Borzi (Borzì, 2006). A somewhat different concept was introduced by A. Dervieux and collaborators who proposed a technique of Hierarchical Preconditioning (Dervieux et al., 2001) in which the multilevel geometrical data structure of agglomeration multigrid is exploited to define a hierarchical optimization algorithm. The major concepts related to this approach have also been presented (Dervieux et al., 2006).

Besides, other types of hierarchical concepts have been introduced in the literature to enhance the efficiency. In particular, certain very efficient optimizers have been developed to employ several models to represent with different degrees of accuracy the same physical situation, the coarsest model having the merit to be very economical to evaluate. In this area, let us cite the concept of low/high-fidelity models in (Anderson et al., 2000; Alexandrov et al., 2001). (Séfrioui, 1998; Séfrioui et $a l ., 2000)$ has adapted a genetic algorithm to introduce a technique of islands in the population of individuals in order to perform a robust working-space exploration at the coarsest level combined with an accurate exploitation at the fine level.

Here, as a sequel of (Désidéri, 2003; Désidéri et al., 2004; Abou El Majd et al., 2005; Désidéri et al., 2006; Désidéri et al., 2007; Désidéri, 2006), we review the construction of multilevel algorithms, in the context of parametric shape optimization. 
Embedded search spaces are defined based on a geometrical hierarchy of nested shape parameterizations of Bézier type. We first consider two-dimensional geometries for which shapes are directly parameterized. Then, we recall the concept of Free-Form Deformation which allows us to extend the multilevel geometrical representation to three-dimensional cases. Then we provide some details on how the usual optimization algorithms (simplex, steepest-descent) can be formulated in the context of a multilevel shape parameterization. We then present results of optimum-shape design in 3D aerodynamics governed by the compressible Euler equations solved by a finite-volume method using unstructured grids. We finally conclude with some perspectives.

\section{Nested Bézier parameterizations for multilevel shape representation}

We begin with the simplest situation of a two-dimensional geometry for which we employ a Bézier shape representation:

$$
x(t)=\sum_{k=0}^{n} B_{n}^{k}(t) x_{k}, \quad y(t)=\sum_{k=0}^{n} B_{n}^{k}(t) y_{k}
$$

in which the parameter $t$ varies from 0 to $1, n$ is the degree of the parameterization,

$$
B_{n}^{k}(t)=C_{n}^{k} t^{k}(1-t)^{n-k}
$$

is a Bernstein polynomial, $C_{k}^{n}=\frac{n !}{k !(n-k) !}$, and

$$
P_{k}=\left(\begin{array}{c}
x_{k} \\
y_{k}
\end{array}\right) \quad(k=0,1, \ldots, n)
$$

is the generic control point. The coordinates of these control points are split into two vectors

$$
X=\left\{x_{k}\right\}, \quad Y=\left\{y_{k}\right\}, \quad k=0,1, \ldots, n,
$$

and we refer to the vector $X$ as the support of the parameterization, and the vector $Y$ as the design vector. Typically, we optimize the design vector for fixed support according to some physical criterion, such as drag reduction in aerodynamics. The somewhat unsymmetrical roles dispensed to the vectors $X$ and $Y$ are chosen to reduce (to $n$ essentially) the dimension of the search space in the optimization phase, which is the most numerically costly and subject to numerical stiffness.

We also use the notation:

$$
x(t)=B_{n}(t)^{T} X, \quad y(t)=B_{n}(t)^{T} Y,
$$

in which the vector $B_{n}(t)^{T}=\left(B_{n}^{0}(t), B_{n}^{1}(t), \ldots, B_{n}^{n}(t)\right)$. In all this article, only supports for which the sequence $\left\{x_{k}\right\}$ is monotone increasing are said to be admissible and considered throughout. Thus, the function $x(t)$ is monotone-increasing and 
defines a one-to-one mapping of, say, [0,1] onto itself. Recall also the simple formula for the derivative:

$$
\frac{d x(t)}{d t}=n \sum_{k=0}^{n-1} B_{n-1}^{k}(t)\left(x_{k+1}-x_{k}\right)=n B_{n-1}(t)^{T} \Delta X
$$

in which $\Delta$ denotes the forward-difference operator $\left(\Delta x_{k}=x_{k+1}-x_{k}\right)$ as well as the associated $n \times(n+1)$ matrix.

In the prototypical case of an airfoil, we use such a parametric representation for both the upper and lower surfaces separately. The vertical slope at the leading edge is enforced by the conditions:

$$
x_{0}=x_{1}=0, \quad y_{0}=0
$$

for both surfaces which assures a smooth match; at the trailing edge, we simply have:

$$
x_{n}=1, \quad y_{n}=0
$$

for a continuous match.

Our geometrical construction employs the degree-elevation process, well-known in the Computer-Aided Design literature (see for example (Farin, 1990)). This process permits to cast [1] into the following equivalent Bézier parameterization of degree $n+1$ :

$$
x(t)=\sum_{k=0}^{n+1} B_{n+1}^{k}(t) x_{k}^{\prime}, \quad y(t)=\sum_{k=0}^{n+1} B_{n+1}^{k}(t) y_{k}^{\prime}
$$

in which the new control points $P_{k}^{\prime}=\left(x_{k}^{\prime}, y_{k}^{\prime}\right)$ are obtained from the former by convex combinations:

$$
P_{0}^{\prime}=P_{0}, P_{k}^{\prime}=\frac{k}{n+1} P_{k-1}+\left(1-\frac{k}{n+1}\right) P_{k}(k=1,2, . ., n), P_{n+1}^{\prime}=P_{n}
$$

obtained by multiplying [1] by $(1-t)+t$ and grouping together the monomials in $t^{k}(1-t)^{n+1-k}$, for each $k$.

Figure 1 represents the RAE2822 airfoil and the lower and upper control polygons of degree-16 Bézier least-squares curvefits. The RAE2822 airfoil is a classical geometry in computational aerodynamics, known for its low-drag performance in the transonic regime. This shape has been tabulated by the European Project ECARP (Périaux et al., 1998).

From a theoretical viewpoint, our construction guarantees rigorously nested search spaces, and exact upward transfer operators (from low to high-degree parameterization). This is illustrated on Figure 2 in which the supports of three nested parameterizations of the airfoil are sketched, without reference to the corresponding sets of ordinates. 


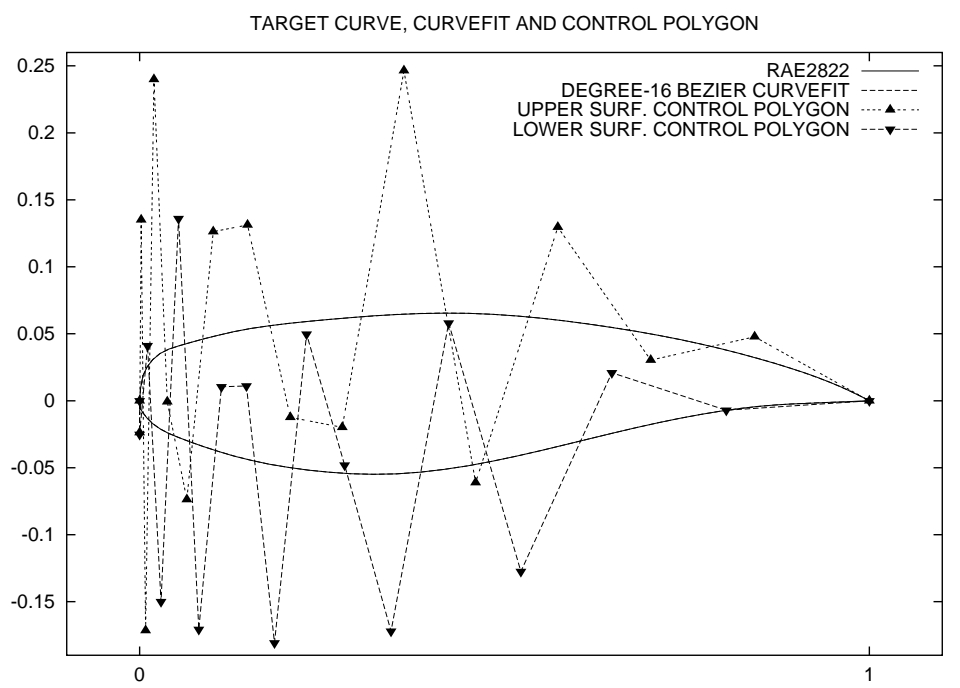

Figure 1. RAE2822 airfoil, examples of degree-16 Bézier curvefi ts of the upper and lower surfaces (superimposed), and corresponding control polygons

(i) $\ell=1, n_{1}=4$ (initial data)

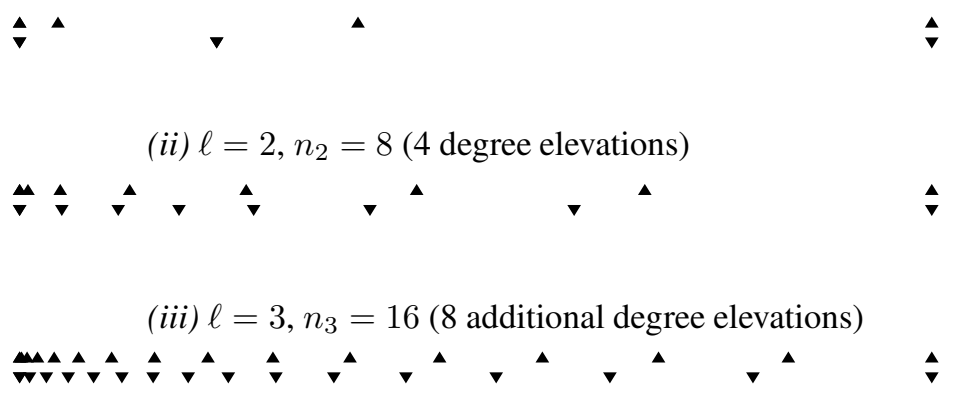

Figure 2. One-dimensional example of embedded parameterizations: the triangles represent the supports $X$ of three nested Bézier parameterizations of degree 4, 8 and 16 of an RAE2822 airfoil obtained from the fi rst by 4 and 12 successive degree elevations; the symbols pointing upward (resp. downward) are associated with the upper (resp. lower) surface; the degree-4 support has been optimized to regularize the control polygon associated with the degree-16 airfoil representation 
Note that in the case of Figure 2, apart from the specified endpoints, the abscissas of the degree-4 support $X$ are not a subset of the abscissas of any support of a higher degree parameterization. Nevertheless, any Bézier curve given on the degree-4 support can be expressed exactly on any other support of higher degree provided it results, as in this example, from the degree elevation process. The parameterizations are nested, or embedded in one another in this sense precisely.

\section{FFD for multilevel three-dimensional shape deformation}

A critical issue in aerodynamic design is the choice of the shape parameterization. Parameterization techniques for practical 3D aerodynamic shape optimization have to fulfill several criteria:

- the parameterization should be able to take into account complex geometries, possibly including constraints and singularities ;

- the number of parameters should be as small as possible, since the stiffness of the shape optimization numerical formulation increases abruptly with the number of parameters ;

- the parameterization should allow to control the smoothness of the resulting shapes.

A survey of shape parameterization techniques for multi-disciplinary optimization, which are analyzed according to the previous criteria, is proposed in (Samareh, 2000). Following his recommendation, conclusions, the Free-Form Deformation (FFD) technique (Sederberg et al., 1986) is adopted in the present study, since it provides an easy and powerful framework for the deformation of complex shapes, such as generic or elaborate aerodynamic configurations.

The FFD technique originates from the Computer Graphics field (Sederberg et al., 1986). It allows the deformation of an object in a 2D or 3D space, regardless of the representation of this object. Instead of manipulating the surface of the object directly, by using classical B-Splines or Bézier parameterization of the surface, the FFD technique defines a deformation field over the space embedded in a lattice which is built around the object. By modifying the space coordinates inside the lattice, the FFD technique deforms the object, regardless of its geometrical description. In particular, the initial geometry, in our applications, is usually defined by a general, Finite-Element-type unstructured simplicial grid.

More precisely, consider a three-dimensional hexaedral lattice embedding the object to be deformed. Figure 3(a) shows an example of such a lattice built around a typical wing. A local coordinate system $(\xi, \eta, \zeta)$ is defined in the lattice, with $(\xi, \eta, \zeta) \in[0,1] \times[0,1] \times[0,1]$. As a result of the deformation, the displacement 
$\Delta q$ of each point $q$ inside the lattice is here defined by a third-order Bézier tensor product:

$$
\Delta q=\sum_{i=0}^{n_{i}} \sum_{j=0}^{n_{j}} \sum_{k=0}^{n_{k}} B_{n_{i}}^{i}\left(\xi_{q}\right) B_{n_{j}}^{j}\left(\eta_{q}\right) B_{n_{k}}^{k}\left(\zeta_{q}\right) \Delta P_{i j k} .
$$

$B_{n_{i}}^{i}, B_{n_{j}}^{j}$ and $B_{n_{k}}^{k}$ are again Bernstein polynomials of order $n_{i}, n_{j}$ and $n_{k}$. $\left(\Delta P_{i j k}\right)_{0 \leq i \leq n_{i}, 0 \leq j \leq n_{j}, 0 \leq k \leq n_{k}}$ are weighting coefficients, or control points displacements, which are used to monitor the deformation and are considered as design variables during the shape optimization procedure. The critical point is that only the shape deformation is represented, not the shape itself.

This technique is illustrated by Figure 3. A lattice is built around a wing and a Bézier tensor product of degree $n_{i}=4, n_{j}=1$ and $n_{k}=1$ is defined over this lattice. Corner control points (filled markers) are supposed to be frozen in order to keep leading and trailing edges fixed during the deformation, whereas other control points (empty markers) are allowed to move vertically (Figure 3(a)). When these control points are moved, their displacements define a continuous deformation inside the lattice according to [11], yielding a shape deformation. The deformed lattice and shape can be seen in Figure 3(b)).

The FFD technique described above is well suited to complex shape optimization, thanks to the following properties:

- the initial shape can be exactly represented (no deformation occurs when all weighting coefficients are zero);

- the deformation is performed whatever the complexity of the shape (this is a free-form technique);

- geometric singularities can be taken into account (the initial shape including its singularities is deformed);

- the smoothness of the deformation is controlled (the deformation is ruled by Bernstein polynomials);

- the number of design variables depends on the user's choice (the deformation is independent of the shape itself);

This technique nicely deals with multilevel representation thanks to the Bézier degree elevation property. To apply the degree elevation process within the FFD framework, one should simply apply the standard degree elevation process for each direction of the tensor product. The process is illustrated by Figure 4. Degree elevation in the direction $\xi$ is applied to the shape obtained previously using a deformation of degree 4-1-1 (Figure 4(a)). Figure 4(b) shows the result when the degree is elevated to 6-11 and Figure 4(c) when it is elevated to 8-1-1. One can notice that the shape is not modified, whereas the lattice counts an increasing number of control points. 


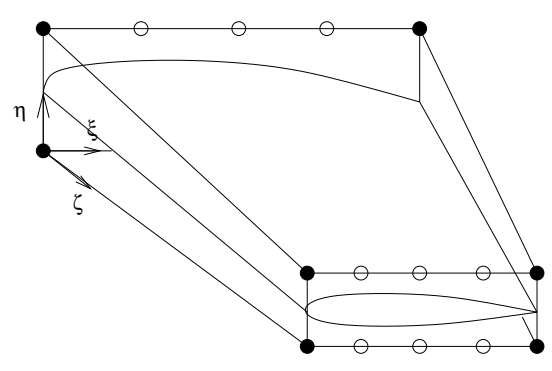

(a) Initial FFD lattice 4-1-1

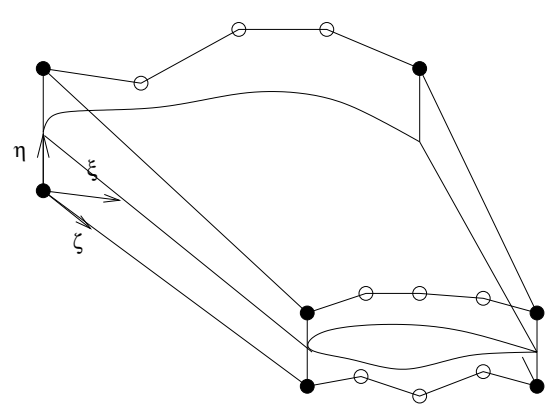

(b) Deformed FFD lattice 4-1-1

Figure 3. Example of Free-Form Deformation: by moving some control points of the lattice, a deformation fi eld is defi ned continuously inside the lattice, yielding a shape deformation

\section{Multilevel variants of classical optimization algorithms}

With the support of the nested geometrical representations constructed in the previous sections, we can now define precisely our algorithms which combine standard iterations (simplex method, particle-swarm optimizer or steepest-descent) with a multilevel geometrical treatment. To be specific, we consider for simplicity the two uniform and nested parameterization supports of Figure 5 associated with representations of shape, or shape-deformation of degree 4 and 8 of a two-dimensional problem. Let $\gamma$ denote the shape to be optimized, and $J(\gamma)$ the intrinsic cost function to be minimized. Let $B_{n}(X, Y)$ denote the Bézier curve of degree $n$ associated with the control points $(X, Y)$. Then, the parametric cost function is taken to be

$$
j_{n}(Y):=J\left(B_{n}(X, Y)\right)
$$

where $X$ is fixed (and possibly subsequently adapted). 


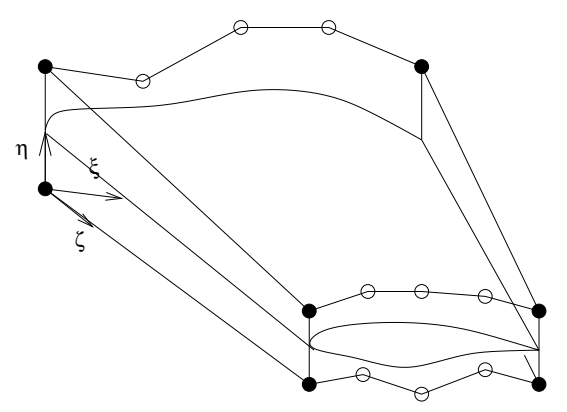

(a) Deformed FFD lattice 4-1-1

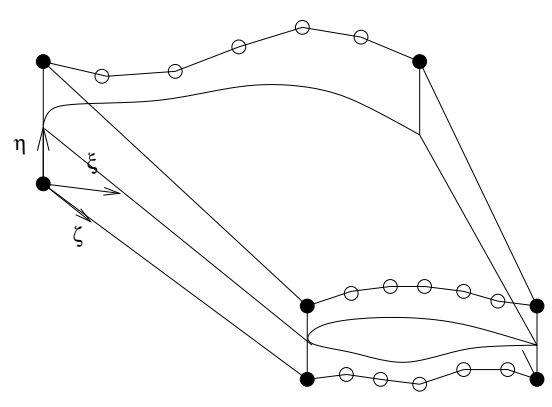

(b) Deformed FFD lattice 6-1-1

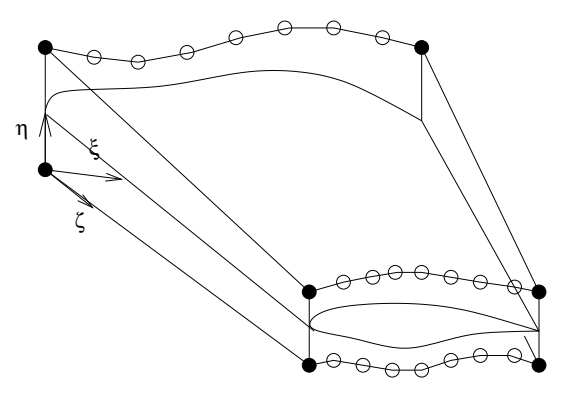

(c) Deformed FFD lattice 8-1-1

Figure 4. Example of degree elevation process: by using the degree elevation process in the direction $\xi$, the number of control points is increased while the initial shape is not modifi ed 


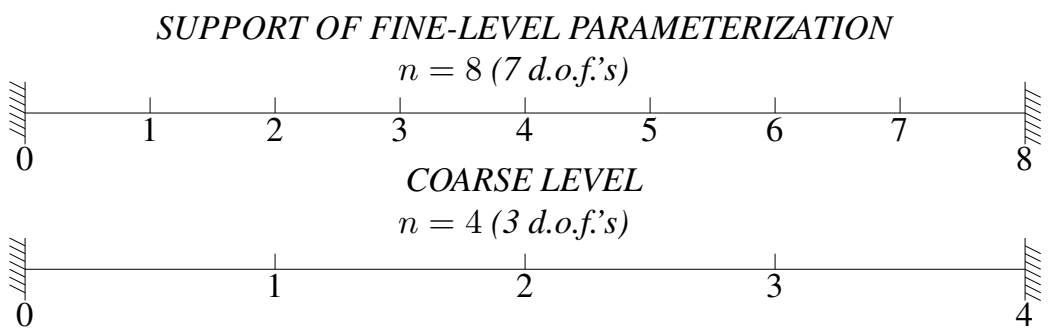

Figure 5. Nested supports of parameterizations of degree 4 and 8; the degrees of freedom associated with both endpoints $(k=0$ or $n)$ are fi xed

Then the classical steepest descent method can be combined with a two-level parameterization in the following algorithm, in which $E_{4}^{8}$ is the rectangular matrix representing the degree-elevation process from $n=4$ to 8 :

1) partially solve coarse-level parametric optimization problem:

$$
\text { For } i=1,2, \ldots, K d o: Y^{\prime i}=Y^{i-1}-\rho_{i} \nabla j_{C O A R S E}\left(Y^{\prime i-1}\right)
$$

Here, $j_{C O A R S E}=j_{4}$ and $Y^{\prime i} \in \mathbb{R}^{5}$ (but with only 3 d.o.f.'s since $y_{0}=y_{4}=0$ )

2) transfer optimum-shape design vector onto upper level

$$
Y^{K}=E_{4}^{8} Y^{\prime K} \quad \Longrightarrow Y^{K} \in \mathbb{R}^{9}
$$

3) solve fine-level parametric optimization problem:

Reset $Y^{0}=Y^{K}$ and for $i=1,2, \ldots$ until convergence:

$$
Y^{i}=Y^{i-1}-\rho_{i} \nabla j_{F I N E}\left(Y^{i-1}\right)
$$

Here, $j_{F I N E}=j_{8}$ and $Y^{i} \in \mathbb{R}^{9}$ (but with only 7 d.o.f.'s since $y_{0}=y_{8}=0$ ) $\Longrightarrow$ final solution $Y^{*} \in \mathbb{R}^{9}$.

In fact, in the above algorithm, all the evaluations are performed on the upper level. Now, since the supports are nested (embedded),

$$
X^{(8)}=E_{4}^{8} X^{(4)}
$$

the above transfer (item 2) is exact:

$$
B_{8}\left(X^{(8)}, Y^{K}\right)=B_{4}\left(X^{(4)}, Y^{\prime K}\right)
$$

and in terms of shape, the upper-level iteration begins with the last update coming from the previous lower-level iteration.

Similarly, the two-level "ideal V-cycle" based on steepest descent is defined by the schematic of Table 1. 
Table 1. Schematic of two-level ideal V-cycle

$$
\begin{aligned}
& \text { Upper level } \\
& \text { For } i=1,2, \ldots, K \text { do : } \\
& Y^{i}=Y^{i-1}-\rho_{i} \nabla j_{F I N E}\left(Y^{i-1}\right) \\
& \Longrightarrow Y^{K} \in \mathbb{R}^{9} \\
& \text { V } \\
& \text { Upper level } \\
& \text { Reset } Y^{0}:=Y^{K}+E_{4}^{8} Y^{\prime *} \text { and do: } \\
& Y^{i}=Y^{i-1}-\rho_{i} \nabla j_{F I N E}\left(Y^{i-1}\right)(i \leq K) \\
& \Longrightarrow Y^{K}:=Y_{\text {new }}^{0} \\
& j_{F I N E}\left(Y^{K}+E_{4}^{8} Y^{\prime}\right) \\
& \Longrightarrow Y^{\prime *} \in \mathbb{R}^{5}
\end{aligned}
$$

Note that above algorithm is said to be ideal if the coarse-level problem is solved to complete convergence regardless the method (evolutionary algorithm, simplex method, steepest-descent, (P)CG, BFGS, etc). Additionally, the iteration is formulated as a correction algorithm since the coarse-level problem defined in terms of a correction vector to be applied to the unknown (upper-level) design vector.

The V-cycle being defined, more elaborate strategies mimicking classical multigrid iterations can be devised. Once the nested supports have been constructed from a coarse parameterization, all multigrid-type strategies (saw-tooth, V or W-cycle, FullMulti-Grid (FMG), (Wesseling, 1992)) can be implemented; at any point in the graph, the current approximate optimum shape admits an exact representation on the fine level.

Note that nested supports of parameterization of shape-deformation are far simpler to construct and handle than nested grids (particularly in the unstructured-grid finite-volume formulation). Consequently, the present multilevel parametric shape optimization algorithms are easy to implement.

In particular, iterations involving all intermediate levels could easily all be considered without particular implementation difficulties.

\section{Application to aerodynamics design}

\subsection{Test-case description}

The test-case considered here corresponds to the optimization of the wing shape of a business aircraft (courtesy of Piaggio Aero Industries) in a transonic regime. The free-stream Mach number is $M_{\infty}=0.83$ and the incidence $\alpha=2^{\circ}$. Initially, the wing section corresponds to the NACA 0012 airfoil. An unstructured mesh, composed of 31124 nodes and 173445 elements, is generated around the wing, including a refined area in the vicinity of the shock (Figure 6). Flow fields are obtained by solving compressible Euler equations using a finite-volume method. 


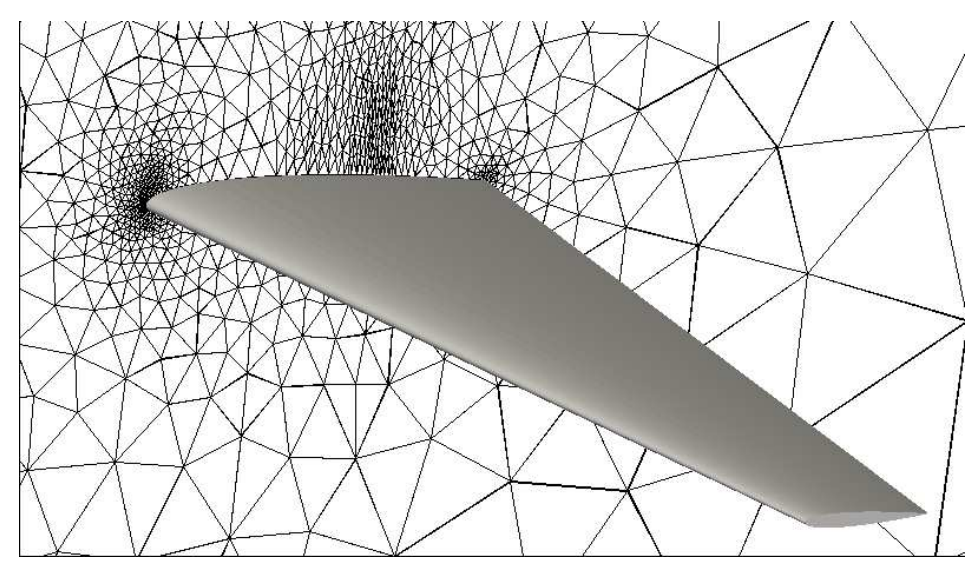

Figure 6. Initial wing shape and mesh in the symmetry plane

The goal of the optimization is to reduce the drag coefficient $C_{D}$ subject to the constraint that the lift coefficient $C_{L}$ should not decrease more than $0.1 \%$. The constraint is taken into account using a penalization approach. Then, the resulting cost function is:

$$
\mathcal{J}_{O P T}=\frac{C_{D}}{C_{D 0}}+10^{4} \max \left(0,0.999-\frac{C_{L}}{C_{L 0}}\right) .
$$

$C_{D_{0}}$ and $C_{L_{0}}$ are respectively the drag and lift coefficients corresponding to the initial shape (NACA 0012 section).

The FFD lattice is built around the wing with $\xi, \eta$ and $\zeta$ in the chord-wise, spanwise and thickness directions respectively. The lattice is chosen in order to fit the planform of the wing. Then, the leading and trailing edges are kept fixed during the optimization by freezing the control points that correspond to $i=0$ and $i=n_{i}$. Moreover, control points are only moved vertically. Results are presented for three parameterizations. The coarsest one corresponds to $n_{i}=3, n_{j}=1$ and $n_{k}=1$. Therefore, $(4-2) \times 2 \times 2=8$ degrees of freedom are taken into account in the optimization. The medium parameterization corresponds to $n_{i}=6, n_{j}=1$ and $n_{k}=1$ and counts $(7-2) \times 2 \times 2=20$ degrees of freedom. Finally, the finest parameterization corresponds to $n_{i}=9, n_{j}=1$ and $n_{k}=1$ and counts $(10-$ 2) $\times 2 \times 2=32$ degrees of freedom. In this study, the Nelder-Mead simplex method (Nelder et al., 1965) is used as optimization algorithm. Three strategies corresponding to different ways of handling the geometrical parameterization are compared:

- basic method (test A): single parameterization until full convergence;

- progressive degree elevation (test B): 3 levels (coarse, medium and fine) are considered successively using the degree elevation transfer;

- FMOSA (test C): using the same 3 levels of parameterization as the ones of test B in a strategy including a FMG-like approach defined in Table 2. 
Table 2. V-cycle fully multilevel algorithm; the parameterization-support adaptions $(\rightarrow)$ are possible at fixed degree prior to degree-elevation+relaxation $\nearrow$ but have not been made in the present tests; again \indicates the formulation of a correction problem over the embedded support inherited from the last adaption; the degrees refer to the parameterization in the vertical direction; the indicated fi gures 10 and 70 correspond to iteration counts

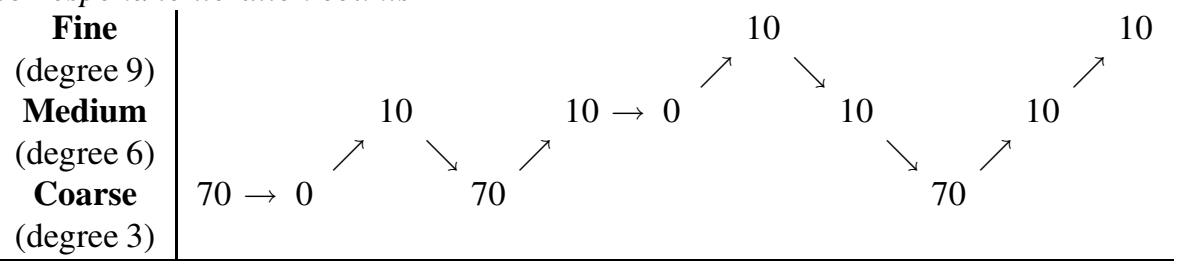

\subsection{Aerodynamic coefficients}

The aerodynamic coefficients obtained for each method are compared in Table 3. The lift coefficient is approximately maintained or slightly increased by the shape optimization process. Important reductions of the drag coefficient are reported. As observed, the multilevel strategies improve significantly the aerodynamic performance.

Table 3. Comparison of aerodynamic coeffi cients and cost function values

\begin{tabular}{|l|c|c|c|c|}
\hline & Method & $C_{L}$ & $C_{D}$ & Cost \\
\hline Reference & & 0.31920 & 0.02635 & 1. \\
\hline Test A & Single fine param. & 0.31978 & 0.1703 & 0.64539 \\
\hline Test B & Degree elev. & 0.31888 & 0.01629 & 0.61823 \\
\hline Test C & FMOSA & 0.31890 & 0.01581 & 0.60027 \\
\hline
\end{tabular}

\subsection{Convergence history plots}

Figure 7 depicts the convergence history of the basic method (single parameterization) for three different parameterizations. With a coarse parameterization, a very fast convergence is observed, but the value of the cost function at convergence is not satisfactory (poor accuracy). Increasing the number of geometrical parameters results in an improved aerodynamic performance, but a larger number of iterations is required. However, one can notice that the optimization using the fine parameterization has not converged yet after 1100 iterations, yielding a performance worse than previously obtained with the medium parameterization.

Figure 8 shows a comparison of the convergence for the three strategies under consideration. The method based on a progressive degree elevation is significantly better than the classical single parameterization approach. FMOSA is still more efficient, yielding a shape of better fitness using a smaller computational effort. 


\subsection{Flows}

A comparison of the flowfields for the final shapes obtained with the different strategies is presented in Figures 9 to 12. The Mach number field on the wing surface and Mach number contours in the symmetry plane are represented. Visibly, this drag reduction exercise results in a strong reduction of the shock wave. Using a single fine parameterization, the shock reduction is not as important, whereas in the multilevel approaches, the shock at the root section disappears.

\section{Conclusions and perspectives}

We have shown in this study that multilevel strategies are better equipped to alleviate the numerical stiffness in numerical shape optimization, which in the parametric formulation increases very rapidly with the number of degrees of freedom (Désidéri, 2006).

Besides, adequate parameterization adaption techniques have been devised and tested in model problems (Désidéri et al., 2007) as well as in three-dimensional flow problems (Duvigneau, 2006). These procedures which readjust the parameterization support to regularize the shape representation, were also found to be very effective in accelerating the convergence process. From a theoretical point of view, we have also proposed (Désidéri, 2006; Désidéri et al., 2006) a shape reconstruction or inverse problem as a simple model to analyze the convergence mechanism of our parametric shape optimization method. There, we have identified a pertinent eigensystem associated with a linear iteration. This analysis comforts us in the analogy with multigrid methods supporting our algorithms, but at the same time, raises a number of differences and theoretical questions requiring further investigation, currently being carried out.

Ultimately, we observe that the multilevel geometrical structure could also be used to support a hierarchical method in which reduced models are used on lower levels. These reduced models can be based on:

- variable physics: e.g. Euler on coarse levels, Navier-Stokes on upper;

- variable numerics: use of a hierarchy of meshes, and/or simplified state-cost functional dependency (response surface, artificial neural networks, etc), and/or alternate optimizer (e.g., evolutionary on coarsest level for robustness, simplex method on intermediate level, deterministic on fine level for accuracy; hybridization), etc.

Thus, a great number of promising algorithmic variants can be devised. 


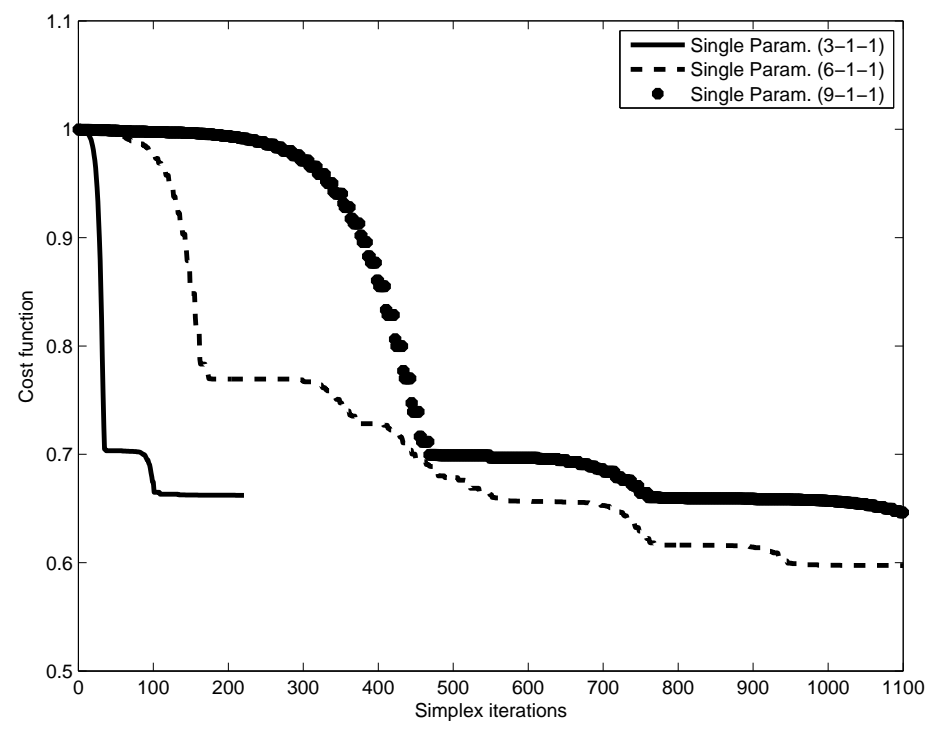

Figure 7. Basic method: convergence history plot for three different single parameterizations

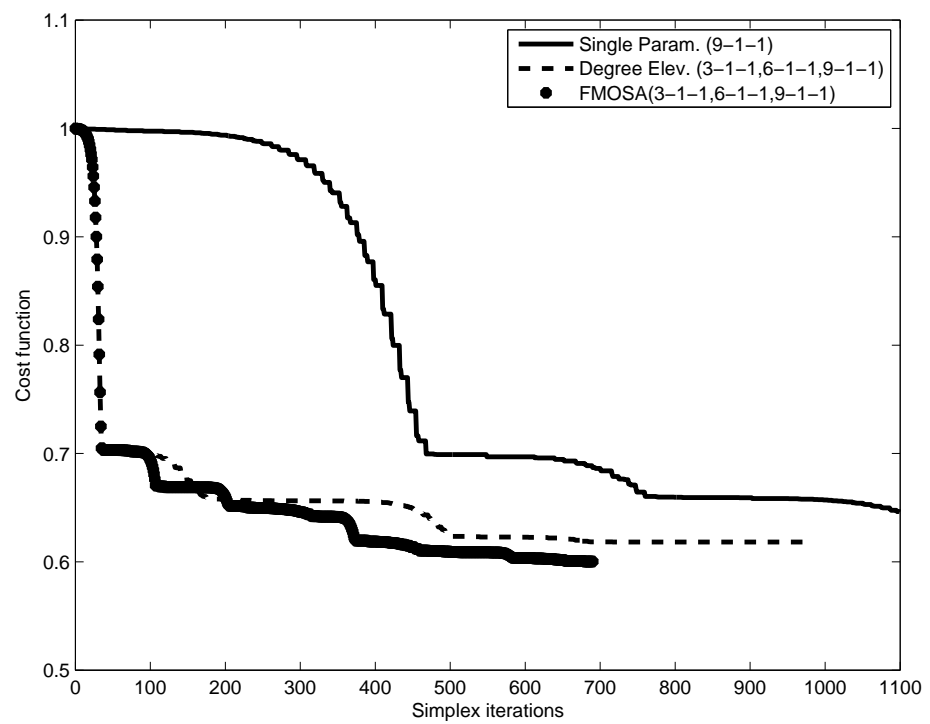

Figure 8. Comparison of the convergence history for the three strategies 


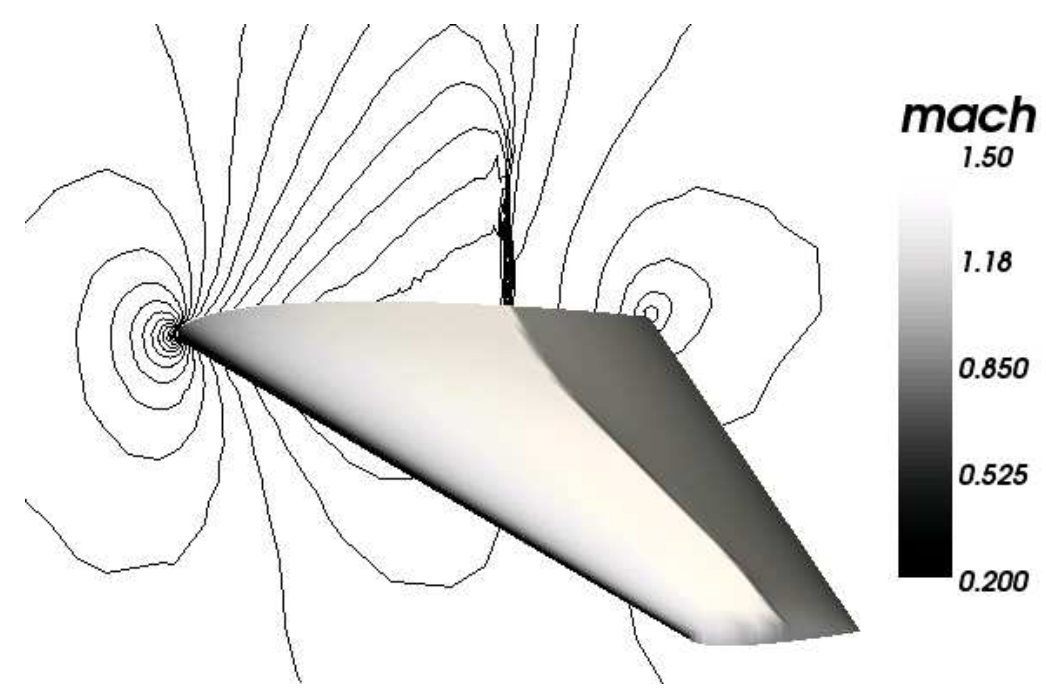

Figure 9. Mach number fi eld on the wing and Mach number contours in the symmetry plane: initial shape

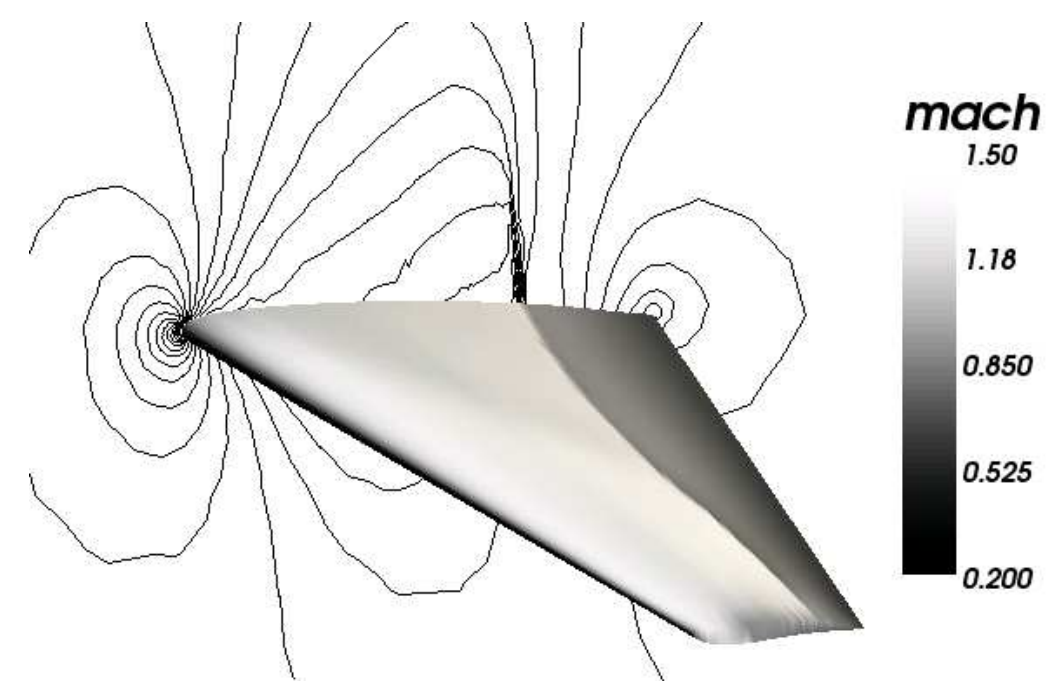

Figure 10. Mach number fi eld on the wing and Mach number contours in the symmetry plane: single fi ne parameterization 


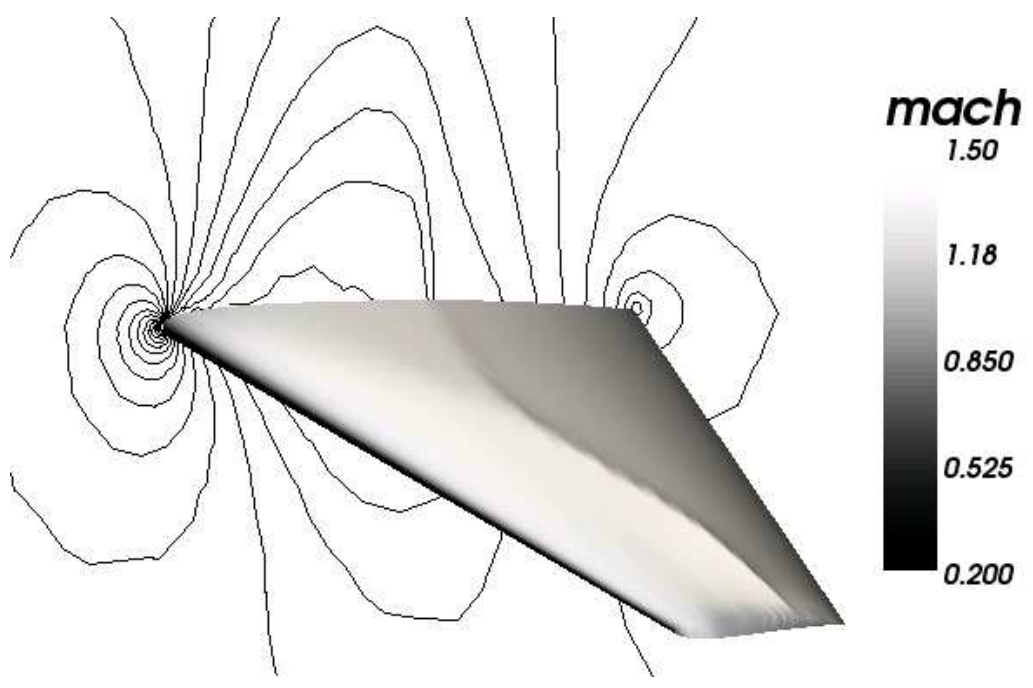

Figure 11. Mach number fi eld on the wing and Mach number contours in the symmetry plane: progressive degree elevation strategy

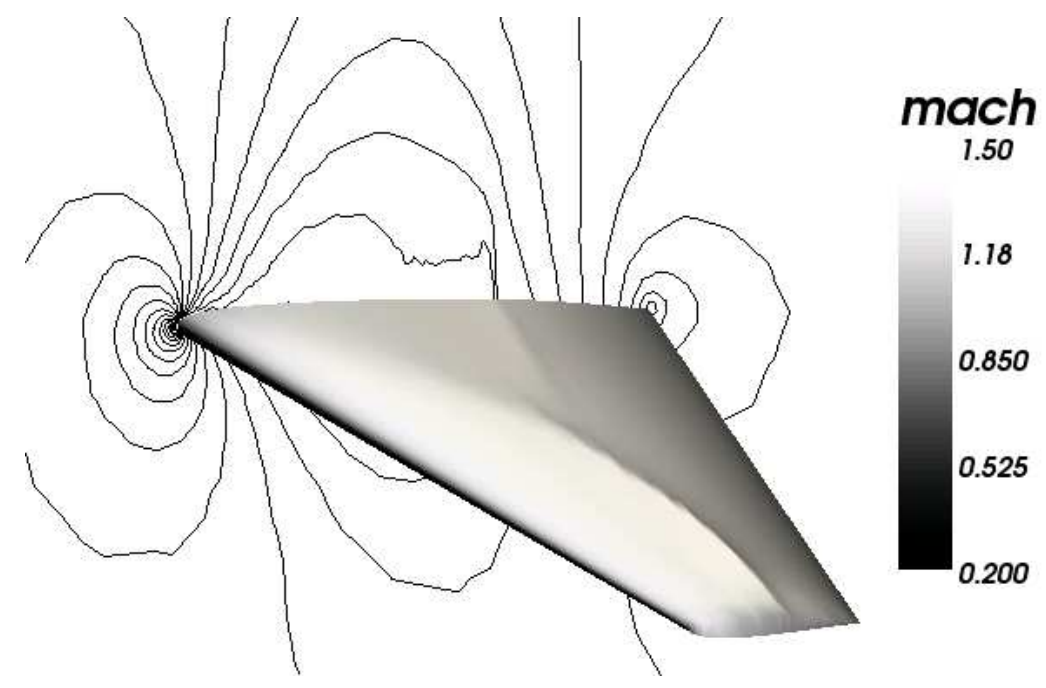

Figure 12. Mach number fi eld on the wing and Mach number contours in the symmetry plane: FMOSA strategy 


\section{References}

Abou El Majd B., Désidéri J.-A., Do T. T., Fourment L., Habbal A., Janka A., “ Multilevel Strategies and Hybrid Methods for Shape Optimization and Applications to Aerodynamics and Metal Forming”, in R. Schilling, W. Haase, J. Périaux, H. Baier, G. Bugeda (eds), Evolutionary and Deterministic Methods for Design, Optimisation and Control with Applications to Industrial and Societal Problems Conference (EUROGEN 2005), Munich, Germany, September 12-14, 2005. (CFLM, Munich, 2005.

Alexandrov N. M., Lewis R. M., Gumbert C. R., Green L. L., Newman P. A., “ Approximation and Model Management in Aerodynamic Optimization with Variable-Fidelity Models", Journal of Aircraft, vol. 38, n 6, p. 1093-1101, 2001.

Anderson W. K., Nielsen E., Alexandrov N., Lewis R., “ First-Order Model Management with Variable Fidelity Physics Applied to Multi-Element Airfoil Optimization”, , AIAA paper 2000-4886, 2000.

Arian E., Ta'asan s., Optimal Design and Control, J. Boggaard, J. Burkardt, M. Gunzburger, J. Peterson Eds., Birkhauser Boston Inc., chapter Shape Optimization in One Shot, 1995.

Borzì A., " Multilevel Methods for Optimization and Inverse Problems", SIAM Annual Meeting 2006, Boston, Minisymposium, 2006. http://www.uni-graz.at/imawww/borzi/.

Dervieux A., Désidéri J.-A., Introduction to Optimization and Multidisciplinary Design, J. Périaux and H. Deconinck Eds., Lecture Series 2006-3, Von Karman Institute for Fluid Dynamics, Belgium, chapter Hierarchical Methods for Shape Optimization in Aerodynamics - II: Additive Methods in Aerodynamics, 2006.

Dervieux A., Marco N., Held C., Koobus B., " Hierarchical Principles and Preconditioning for Optimum Design and Identification”, in J. P. et al (ed.), Innovative Tools for Scientific Computation in Aeronautical Engineering, Handbooks on Theory and Engineering Applications of Computational Methods, CIMNE, Barcelona, 2001.

Désidéri J.-A., Numerical Methods for Scientific Computing, Variational Problems and Applications, E. Heikkola, Y. Kuznetsov, P. Neittaanmäki and O. Pironneau eds., CIMNE, chapter Hierarchical Optimum-Shape Algorithms Using Embedded Bézier Parameterizations, 2003.

Désidéri J.-A., “ Two-Level Ideal Algorithm for Parametric Shape Optimization”, in W. Fitzgibbon, R. Hoppe, J. Périaux, O. Pironneau, Y. Vassilevski (eds), Advances in Numerical Mathematics, p. 65-85, n.d. Proc. of two International Conferences: Moscow, Institute of Numerical Mathematics, Russian Academy of Sciences, Sept. 16-17, 2006 and Houston, 2006; in press.

Désidéri J.-A., Abou El Majd B., Janka A., “ Nested and Self-Adaptive Bézier Parameterizations for Shape Optimization”, J. Comput. Phys., 2007. to appear.

Désidéri J.-A., Dervieux A., Introduction to Optimization and Multidisciplinary Design, J. Périaux and H. Deconinck Eds., Lecture Series 2006-3, Von Karman Institute for Fluid Dynamics, chapter Hierarchical Methods for Shape Optimization in Aerodynamics - I: Multilevel Algorithms for Parametric Shape Optimization, 2006.

Désidéri J.-A., Janka A., “ Multilevel Shape Parameterization for Aerodynamic Optimization - Application to Drag and Noise Reduction of Transonic/Supersonic Business Jet”, in P. Neittaanmäki, T. Rossi, S. Korotov, E. Onate, J. Périaux, D. Knörzer (eds), European Congress on Computational Methods in Applied Sciences and Engineering, ECCOMAS 2004, Jyväkyla, Finland, 24-28 July, 2004. 
Duvigneau R., Adaptive Parameterization Using Free-Form Deformation for Aerodynamic Shape Optimization, Research Report $\mathrm{n}^{\circ}$ 5949, INRIA, 2006.

Farin G., Curves and Surfaces for Computer-Aided Geometric Design - A Practical Guide, W. Rheinboldt and D. Siewiorek eds., Academic Press, Boston, 1990.

Haase W., et al (eds), ERCOFTAC Introductory Course to Design Optimisation, Manchester, $U K$, April 6-8, 2005.

Jameson A., “ Aerodynamic Design via Control Theory”, Journal of Scientific Computing, vol. 3, n 97-0101, p. 233-260, 1988.

Jameson A., Martinelli L., Pierce N. A., “ Optimum Aerodynamic Design Using the NavierStokes, Equation", Theorical and Computational Fluid Dynamics, vol. 10, p. 213-237, 1998.

Lewis R., Nash S., “ A Multigrid Approach to the Optimization of Systems Governed by Differential Equations", American Institute of Aeronautics and Astronautics, Reston, VA, $\mathrm{n}^{\circ}$ AIAA-2000-4890, 2000.

Lewis R., Nash S., “ Model Problems for the Multigrid Optimization of Systems Governed by Differential Equations”, SIAM J. Sci. Comput., vol. 26, nº 6, p. 1811-1837, 2005.

Nelder J. A., Mead R., “ A Simplex Method for Function Minimization”, Computer Journal, vol. 7, p. 308-313, 1965.

Périaux J., Bugeda G., Chaviaropoulos P. K., Giannakoglou K., Lantéri S., Mantel B. (eds), Optimum Aerodynamic Design \& Parallel Navier-Stokes Computations, ECARP European Computational Aerodynamics Research Project, Notes on Numerical Fluid Mechanics, Vieweg, Braunschweig/Wiesbaden, Germany, 1998.

Périaux J., Deconinck H. (eds), Introduction to Optimisation and Multidisciplinary Design, Lecture Series 2006-03, Von Karman Institute for Fluid Dynamics, 2006.

Samareh J. A., “ Multidisciplinary Aerodynamic-Structural Shape Optimization Using Deformation (MASSOUD)", 8th AIAA/NASA/USAF/ISS, MO Symposium on Multidisciplinary Analysis and Optimization, September 6-8, 2000/Long Beach, CA, $\mathrm{n}^{\circ} 4911$ in AIAA-2000, 2000.

Sederberg T., Parry S., “ Free-From Deformation of Solid Geometric Models”, Computer Graphics, vol. 20, $\mathrm{n}^{\circ}$ 4, p. 151-160, 1986. See also: Computer Aided geometric Design, Sederberg, T.W., http://tom.cs.byu.edu/ tom/ (Item: Courses).

Séfrioui M., Algorithmes évolutionnaires pour le calcul scientifique : Application à l'électromagnétisme et à la mécanique des fluides, $\mathrm{PhD}$ thesis, University of Paris 6, LIP6, 1998.

Séfrioui M., Périaux J., “ A Hierarchical Genetic Algorithm Using Multiple Models for Optimization", Proceedings of the 6th International Conference on Parallel Problem Solving from Nature, Springer-Verlag, London, UK, p. 879-888, 2000.

Wesseling P., An Introduction to Multigrid Methods, John Wiley \& Sons Ltd, 1992. Corrected Reprint: Philadelphia, R.T. Edwards, Inc., 2004.

Winter-Althaus G., et al (eds), ERCOFTAC Design Optimisation: Methods \& Applications, Las Palmas de Gran Canaria, Spain, April 5-7, 2006. 